instance' and only if 'local measures have proved ineffective or there is evidence of cellulitis, spreading infection or systemic involvement' should an antibiotic be prescribed. ${ }^{3}$ Indeed, in the majority of cases of dentoalveolar abscesses, drainage and removal of the source of infection are the only treatments required. ${ }^{4}$

Failing to attempt a surgical procedure (be it extraction, endodontic treatment or soft tissue incision and drainage) when a patient presents with a dentoalveolar abscesses represents a missed opportunity to deal with the cause of the infection. Furthermore, prescribing antibiotics and delaying dental extraction risks the patient developing a potentially serious head and neck infection. Inappropriate use of antibiotics also contributes to the emergence of antibiotic resistant bacterial colonies within the worldwide community, which has become one of the most pressing public health threats of our time. When you consider that dentists in England and Wales are responsible for the prescription of 9\% of all primary care antibiotics, ${ }^{5,6}$ it becomes clear that we as dental practitioners have an important role to play in the responsible use of these drugs.

We therefore applaud Dr Williams' attitude to the management of this and other patients and would encourage other colleagues to do the same: always attempt local, operative measures when faced with odontogenic infections.

A. Cope, I. G. Chestnutt, N. Francis, Cardiff

1. Williams P R. Fat faces and swellings. Br Dent J 2013; 214: 48.

2. Cope A, Francis N, Wood F, Mann M K, Chestnutt I G. Systemic antibiotics for symptomatic apical periodontitis and acute apical abscess in adults. Cochrane Database Syst Rev 2012; 10

3. Scottish Dental Clinical Effectiveness Programme. Drug prescribing for dentistry: dental clinical guidance. Dundee: Scottish Dental Clinical Effectiveness Programme, 2011. Available at www.sdcep. org.uk/?o=2334.

4. Ellison S J. The role of phenoxymethylpenicillin amoxicillin, metronidazole and clindamycin in the management of acute dentoalveolar abscesses - a review. Br Dent J 2009; 206: 357-362.

5. Karki A J, Holyfield G, Thomas D. Dental prescribing in Wales and associated public health issues. BrDent J 2011; 210: E21.

6. The NHS Information Centre, Prescribing Support and Primary Care Services. Prescribing by dentists. England - 2011. London: The Health and Social Care Information Centre, Prescribing Support Unit, 2012.

DOI: 10.1038/sj.bdj.2013.282

\section{MONETARY METAPHOR}

Sir, 'Purse with teeth', eh? (BDJ cover, volume 214 issue 2). I think we can do better than that! As soon as she saw the artwork on the cover our dental nurse immediately came up with an alternative: snappy, memorable, a metaphor for our times: 'Ah,' she exclaimed. 'Money talks!' Well done, Jenny!

J. Le Couteur, Featherstone DOI: $10.1038 /$ sj.bdj.2013.283

\section{COSMETIC TONGUE SPLIT}

Sir, a 30-year-old male attended the ActE department at Whipps Cross Hospital complaining of temperature, severe pain, swelling and difficulty eating, swallowing and bad taste following the cosmetic split of his tongue by a tattooist. Examination revealed a bifid tongue (Fig. 1). The cut edges were approximated with a cotton threadlike material, swollen, erythematous and tender to touch. The patient was pyrexic with elevated blood pressure. Local anaesthesia was infiltrated into the tongue; the 'sutures' were removed, the tongue cleaned with betadine and the edges re-approximated using absorbable sutures. The patient was admitted overnight for intravenous antibiotics and steroids and discharged the following day.

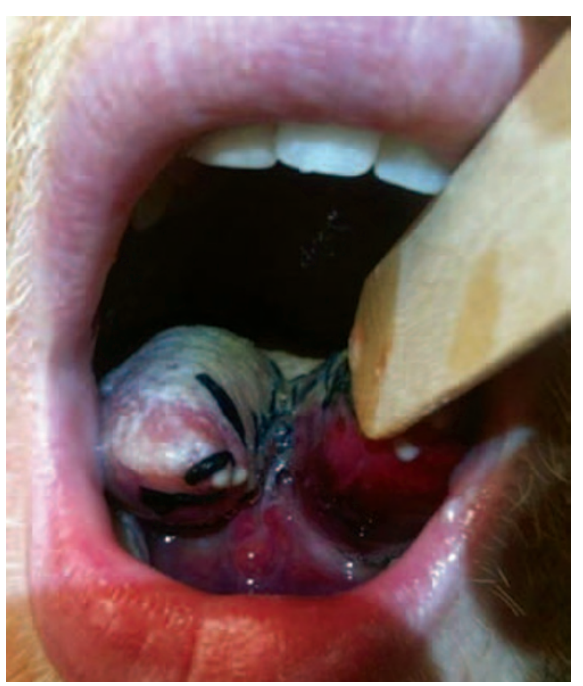

Fig. 1 Bifid tongue on presentation

All surgery carries risks but in the case of tongue surgery, specific risks include bleeding, swelling, lingual nerve damage, infection, scarring and speech distortion. ${ }^{1}$ Failure to assess medical history prior to performing such surgery could have morbid consequences. Sterilisation of equipment in an autoclave and disposable gloves are necessary to avoid transmission of infections such as hepatitis and HIV. ${ }^{2}$ Psychological evaluation of patients wishing to undergo body modification or mutilation procedures is also an important part of pre-operative assessment to identify disorders such as body dysmorphic disorder.

When a random sample of tattooists in the East London area were telephoned and asked if they carried out tongue splitting, none of them alleged that they were licensed to perform it. We contacted the General Medical Council, who advised that the procedure carried out by the tattooist was not classed as 'surgery' as it was carried out for 'cosmetic rather than health reasons'. They stated that they are solely responsible for regulating registered medical professionals and had no jurisdiction over tattooists.

There is no minimum formal training requirement for tattooists, and no clear laws preventing a tattooist from carrying out a cosmetic tongue splitting procedure. Consumers have protection under general consumer law if they are dissatisfied, and may report tattooists to local authority enforcement officers. ${ }^{3}$

Healthcare professionals should consider petitioning the government to introduce regulation and registration for tattoo artists and banning procedures that involve cutting or stitching by non-medical professionals.

F. Aga, R. Harris By email

1. Bressmann T. Self-inflicted cosmetic tongue split: a case report. J Can Dent Assoc 2004; 70: 156-157.

2. Fleming PS, Flood T R. Bifid tongue - a complication of tongue piercing. Br Dent J 2005; 198: 265-266.

3. Smith L. Regulation of tattooing and body piercing businesses. House of Commons Library Science and Environment Section SN/SC/5079, 2010.

DOI: 10.1038/sj.bdj.2013.284

The $B D J$ website now includes a facility enabling readers to immediately comment on letters. All comments must comply with the nature.com Terms and Conditions and Community Guidelines visit the $B D J$ website to find out more and to post your comment now. 